\title{
Correction to: Assessing Electronic Properties of Desymmetrized Heterocyclic Patterns: Towards Tuning Small Molecules for Photovoltaic Applications
}

\author{
Oscar González-Antonio ${ }^{1}$ - Rebeca Yépez ${ }^{2}$ - María Magdalena Vázquez-Alvarado ${ }^{1}$ - Blas Flores-Pérez ${ }^{1}$. \\ Norberto Farfán ${ }^{1} \cdot$ Carlos Amador-Bedolla ${ }^{3} \cdot$ Margarita Romero-Ávila $^{1} \cdot$ Rosa Santillan $^{2}$
}

Published online: 25 March 2021

(c) The Author(s), under exclusive licence to The Materials Research Society 2021

\section{Correction to: \\ MRS Advances volume 5, pages 3171-3184 (2020) \\ https://doi.org/10.1557/adv.2020.434}

This article was updated to correct Rosa Santillan's affiliation.

The original article can be found online at https://doi.org/10.1557/ adv.2020.434.

Norberto Farfán

norberto.farfan@gmail.com

$\bowtie$ Margarita Romero-Ávila

mago_ro@msn.com

$\triangle$ Rosa Santillan

rsantill@ cinvestav.mx

1 Facultad de Química, Departamento de Química Orgánica, Universidad Nacional Autónoma de México. Ciudad

Universitaria, 04510 Ciudad de México, México

2 Departamento de Química, Centro de Investigación y de Estudios Avanzados del IPN, CINVESTAV. Apdo. Postal 14-740, 07000 Ciudad de México, México

3 Facultad de Química, Departamento de Física y Química Teórica, Universidad Nacional Autónoma de México. Ciudad Universitaria, 04510 Ciudad de México, México 